\title{
Geometric phase effects in $\mathrm{H}_{3}$ predissociation
}

\author{
Bruno Lepetit* \\ Laboratoire Collisions, Agrégats, Réactivité (UMR 5589, CNRS and Université Paul Sabatier Toulouse 3) IRSAMC, \\ 31062 Toulouse Cedex 9, France \\ Ravinder Abrol and Aron Kuppermann \\ Division of Chemistry and Chemical Engineering, California Institute of Technology, Pasadena, California 91125, USA
}

(Received 1 June 2007; published 19 October 2007)

\begin{abstract}
We model the predissociation of $\mathrm{H}_{3}$ in the electronic state corresponding to the upper sheet of the conically intersecting $1^{2} A^{\prime}$ and $2^{2} A^{\prime}$ states, and we show that product-state rovibrational distributions are strongly influenced by the geometric phase. Similarly, the differences in the product-state energy distributions in recent three-body dissociation experiments for the $2 s,{ }^{2} A_{1}^{\prime}$ and $2 p,{ }^{2} A_{2}^{\prime \prime}$ states of $\mathrm{H}_{3}$ are shown to result from the presence of the geometric phase in this system, and thus provide experimental evidence of the influence of this phase in a molecular dynamical process. DOI: 10.1103/PhysRevA.76.040702

PACS number(s): 34.50.Pi, 34.10.+x
\end{abstract}

Rydberg states of $\mathrm{H}_{3}$, which play an important role in the dissociative recombination of $\mathrm{H}_{3}^{+}$[1], were first observed by Herzberg [2] in the visible light emission spectra induced by electric discharges through $\mathrm{H}_{2}$. These spectra were understood on the basis of a model involving an active electron interacting with the $\mathrm{H}_{3}{ }^{+}$ionic core [3-6]. In this model, the $\mathrm{H}_{3}$ states are labeled by the principal quantum number $n$ and the orbital angular momentum $L$ of the active electron, in addition to the symmetry of the state with respect to permutation of the identical $\mathrm{H}$ nuclei and parity with respect to inversion of all coordinates. These Rydberg states are all embedded in the continuum and predissociate with lifetimes shorter than $1 \mu \mathrm{s}$. Their predissociation has been the subject of intense experimental studies through coincidence measurements of the fragments, for two-body $[7,8]$ as well as three-body [8-12] dissociation.

The two lowest of these states labeled $2 p,{ }^{2} E^{\prime}$ display a Jahn-Teller effect: they are degenerate for equilateral triangular configurations of the nuclei at which the corresponding adiabatic sheets $1^{2} A^{\prime}$ and $2^{2} A^{\prime}$ conically intersect [13]. The $2^{2} A^{\prime}$ upper sheet can support quasibound states (cone states) [14] which predissociate by vibronic couplings to the lower sheet. The presence of the conical intersection induces a geometric phase, a particular case of Berry's phase [15], on the adiabatic electronic wave functions, namely, a change of their signs when performing a closed loop around the conical intersection [16] in configuration space. Whereas the impact of this phase has been observed on several molecular spectra (Ref. [17] and references therein), there is up to now no evidence of the effect of this phase on dynamical processes. For instance, calculations predict limited effects on the $\mathrm{H}$ $+\mathrm{H}_{2}$ reaction differential cross sections [18], which still await experimental confirmation. The first aim of the present Rapid Communication is to show that this phase can impact significantly product-state distributions of $\mathrm{H}_{3}$ and $\mathrm{D}_{3}$ predissociation, from their $2^{2} A^{\prime}$ cone states for which accurate numerical simulations can be performed. The second aim of this Rapid Communication is to show that the recent mea-

\footnotetext{
*bruno.lepetit@irsamc.ups-tlse.fr
}

surements of product-state energy distributions in three-body dissociation experiments $[11,12]$ for the $2 s,{ }^{2} A_{1}^{\prime}$ and $2 p,{ }^{2} A_{2}^{\prime \prime}$ states of $\mathrm{H}_{3}$ and $\mathrm{D}_{3}$ can be interpreted as being due to the presence of the geometric phase in this system.

We now present our model for the two-body predissociation of the $2^{2} A^{\prime}$ cone states. Nuclear spin does not play a significant role in the physical processes considered here, and the electronuclear wave functions used do not include it. They are called rovibronic wave functions because they include electronic, vibrational, and rotational contributions. We use the DSP diabatic electronic potential energy surfaces $[19,20]$, designed to be particularly accurate in the vicinity of the conical intersection. In the strong-interaction region where reaction takes place, we use row-orthonormal hyperspherical coordinates [21] to describe the system of three atoms in a body-fixed frame $(X, Y, Z)$ tied to the principal axes of inertia of the system. In the weak-interaction region, we use symmetrized hyperspherical coordinates [21], and in the asymptotic region, Jacobi coordinates, to describe the two fragments. The scattering wave function is obtained by an expansion in surface functions, which are solutions of the fixed hyperradius bound-state problem solved using a hyperspherical harmonics basis set $[22,23]$. The scattering and Smith lifetime [24] matrices are extracted from the asymptotic form of the wave function. In the vicinity of a resonance, one eigenvalue of the Smith lifetime matrix is a Lorentzian, whose maximum gives the lifetime of the resonance, and the corresponding eigenvector provides the product-state distribution of the resonance decay. We perform two sets of calculations, both for zero total angular momentum. The first involves expansion of the rovibronic wave function in the pair of coupled electronic diabatic states of symmetry $2 p,{ }^{2} E^{\prime}$. In order to extract the electronically nonadiabatic effects, we compare it with a second set performed with the single adiabatic $1^{2} A^{\prime}$ ground state including the geometric phase [25]. We consider rovibronic wave functions belonging to the $A_{1}$ or $A_{2}$ irreducible representation of the permutation group $P_{3}$ of the identical nuclei.

Smith lifetime matrices for calculation of the pair of coupled diabatic states differ from the single adiabatic state results mainly by the addition of Lorentzian-shaped eigen- 


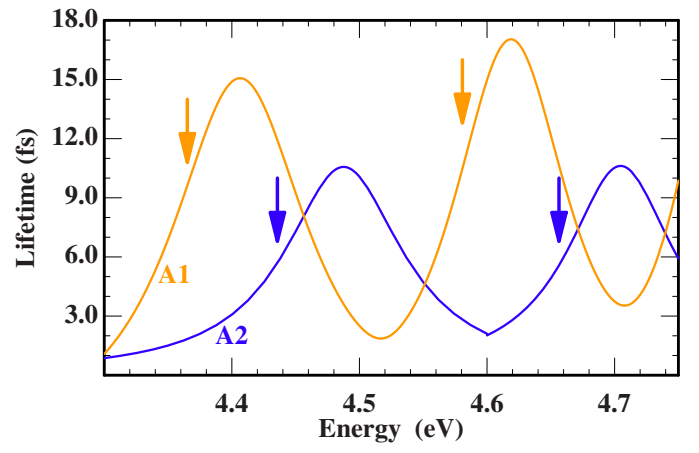

FIG. 1. (Color online) Smith lifetimes for $A_{1}$ and $A_{2}$ rovibronic wave functions. The vertical arrows indicate the energies of the bound states on the upper electronic potential energy surface, when coupling to the ground electronic state is neglected.

values near and above $4 \mathrm{eV}$. These extra eigenvalues are shown as a function of energy for both $A_{1}$ and $A_{2}$ symmetries of the rovibronic wave function in Fig. 1. There are two maxima near 4.41 and $4.62 \mathrm{eV}$ for $A_{1}$ symmetry, and 4.49 and $4.70 \mathrm{eV}$ for $A_{2}$ symmetry. They correspond to resonances with lifetimes close to $15 \mathrm{fs}$ for $A_{1}$ symmetry and $10 \mathrm{fs}$ for $A_{2}$ symmetry. Resonances with similar lifetimes have been computed in Refs. [26,27] and detected experimentally in Ref. [28].

Figure 2 shows the product-state distributions after decay of the $A_{1}$ and $A_{2}$ resonances at 4.41 and $4.49 \mathrm{eV}$, respectively. In both cases, $\mathrm{H}+\mathrm{H}_{2}$ decay products have significant internal energy: for the $A_{1}$ symmetry, $41 \%$ of the available energy goes into rovibrational energy, and $51 \%$ for the $A_{2}$ case. Thus, these resonances decay exclusively into excited rovibrational states and were not observed in previously computed reactive scattering transition probabilities and cross sections $[29,30]$, which were performed for low excitation either of the reactants or of the products. In our reaction probabilities, these resonances display significant intensities only for transitions between simultaneously excited reactants and products.

Internal energy partitioning between vibration and rotation is very different for $A_{1}$ and $A_{2}$ symmetries: $18 \%$ of the internal energy goes into rotation for the $A_{1}$ symmetry, in contrast with $50 \%$ for the $A_{2}$ symmetry. This reflects itself in the product state distributions of Fig. 2, which have a maximum for low rotational quantum number $j$ in the $A_{1}$ symmetry, but for $j$ near 15 for the $A_{2}$ case.

Interpretation of these results requires a knowledge of the nuclear permutation symmetry of the vibrational wave function, which is strongly influenced by the presence of the geometric phase. This phase appears for a pseudorotation $\mathcal{D}$ of the system, starting at an obtuse (principal vertex angle larger than $60^{\circ}$ ) isosceles triangular configuration, formed by the three atoms $1-3$, atom 1 being at the principal vertex. $\mathcal{D}$ is defined to allow atom 1 to move on a circle centered on the vertex of the equilateral triangular configuration and finally to return to its initial position. Application of $\mathcal{D}$ keeps the rovibronic wave function unchanged, but the signs of its electronic, vibrational, or rotational parts may (antiperiodic boundary condition) or may not (periodic boundary condition) change. For instance, $\mathcal{D}$ induces a sign change in the
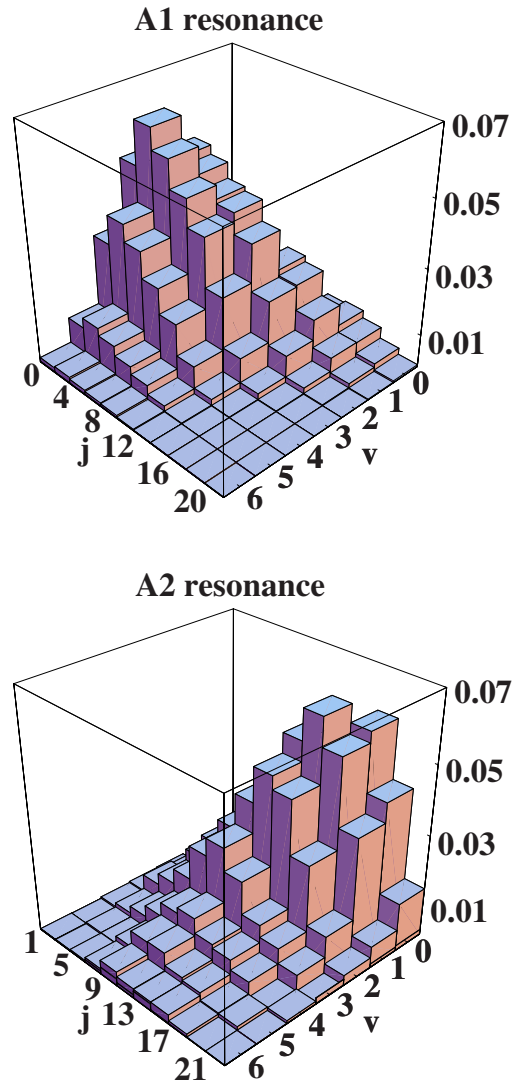

FIG. 2. (Color online) Population of the different $\mathrm{H}+\mathrm{H}_{2}(v, j)$ channels in the decay of the $A_{1}$ and $A_{2}$ resonances at 4.41 and $4.49 \mathrm{eV}$, respectively.

adiabatic $1^{2} A^{\prime}$ and $2^{2} A^{\prime}$ electronic wave functions [16], but not in the $2 s,{ }^{2} A_{1}^{\prime}$ and $2 p,{ }^{2} A_{2}^{\prime \prime}$ ones. Applying $\mathcal{D}$ also induces a flip in the orientation of two of the principal axes of inertia [21], which may induce a sign change of the rotational wave function, according to the value of the total angular momentum. For periodic boundary conditions, the electronic, vibrational, or rotational wave functions also have an $A_{1}$ or $A_{2}$ symmetry. $A_{1}$ vibrational wave functions have no nodal surface prescribed by nuclear permutation symmetry, whereas $A_{2}$ vibrational wave functions have nodal surfaces imposed by nuclear permutation symmetry for both acute (principal vertex angle smaller than $60^{\circ}$ ) and obtuse isosceles configurations. For the antiperiodic boundary condition case, we use the irreducible representations of the double group associated to $P_{3}$ [31]. We call $\bar{A}_{1}\left(\bar{A}_{2}\right)$ the one-dimensional irreducible representations which are symmetric (antisymmetric) with respect to the permutation of atoms 2 and 3. The $1^{2} A^{\prime}$ and $2{ }^{2} A^{\prime}$ adiabatic electronic wave functions have $\bar{A}_{1}$ and $\bar{A}_{2}$ permutational symmetries, respectively. $\bar{A}_{1}\left(\bar{A}_{2}\right)$ vibrational wave functions have nodal planes for obtuse (acute) isosceles configurations and maxima for acute (obtuse) configurations, respectively [21,32]. Loosely speaking, $\bar{A}_{1}$ and $\bar{A}_{2}$ vibrational wave functions therefore have dominant acute and obtuse isosceles characters, respectively.

The energies of the two lowest bound states on the $2^{2} \mathrm{~A}^{\prime}$ upper electronic sheet are shown in Fig. 1 for $A_{1}$ and $A_{2}$ rovibronic symmetries. The corresponding vibrational wave 
TABLE I. Symmetries of the electronic, vibrational, and rotational parts of the rovibronic wave functions in the predissociation of $2{ }^{2} A^{\prime}$ (first pair of rows), $2 p,{ }^{2} A_{2}^{\prime \prime}$ (second pair), and $2 s,{ }^{2} A_{1}^{\prime}$ (third pair) states. The first row of each pair corresponds to the symmetries of the quasibound initial state and the second row to those of the final continuum state. $N$ is the total angular momentum, $K$ its projection on the axis perpendicular to the molecular plane, and $l$ the vibrational angular momentum. Rovibronic symmetries are the products of electronic, vibrational, and rotational symmetries.

\begin{tabular}{lcccc}
\hline \hline & Electronic & Vibrational & Rotational & Rovibronic \\
\hline $2{ }^{2} A^{\prime}(l=3 / 2, N=0, K=0)$ & $\bar{A}_{2}$ & $\bar{A}_{2} / \bar{A}_{1}$ & $A_{1}$ & $A_{1} / A_{2}$ \\
$1{ }^{2} A^{\prime}(N=0, K=0)$ & $\bar{A}_{1}$ & $\bar{A}_{1} / \bar{A}_{2}$ & $A_{1}$ & $A_{1} / A_{2}$ \\
\hline $2 p,{ }^{2} A_{2}^{\prime \prime}(l=0 ; N=0,2 ; K=0)$ & $A_{2}$ & $A_{1}$ & $A_{1}$ & $A_{2}$ \\
$2{ }^{2} A^{\prime}(N=2 ; K= \pm 1)$ & $\bar{A}_{2}$ & $A_{1}$ & $\bar{A}_{1}$ & $A_{2}$ \\
\hline $2 s,{ }^{2} A_{1}^{\prime}(l=0 ; N=1,3 ; K=0)$ & $A_{1}$ & $A_{1}$ & $A_{2}$ & $A_{2}$ \\
$2{ }^{2} A^{\prime}(N=1,3 ; K=0)$ & $\bar{A}_{2}$ & $\bar{A}_{2}$ & $A_{2}$ & $A_{2}$ \\
\hline \hline
\end{tabular}

functions have $\bar{A}_{2}$ and $\bar{A}_{1}$ symmetries (see Table I) and a vibrational angular momentum $l=3 / 2$ [14]. There is a clear correlation between resonance and bound-state energies, with a small shift of the order of $0.05 \mathrm{eV}$. This correlation allows us to characterize the resonances as quasibound cone states predissociating through electronic nonadiabatic couplings to the lower sheet. These resonances are examples of Slonczewski excited states [33], stabilized by the vibrational angular momentum $l=3 / 2$ which creates a pseudocentrifugal barrier pushing the vibrational wave function away from the conical intersection region where electronic nonadiabatic coupling is strongest. The final continuum $A_{1}$ and $A_{2}$ rovibronic wave functions in the ground $1^{2} A^{\prime}$ electronic state have $\bar{A}_{1}$ and $\bar{A}_{2}$ vibrational symmetries (Table I), which have, respectively, acute and obtuse isosceles dominant characters. For near acute isosceles configurations, the decay mechanism is an abstraction one, in which one atom departs from the two others without providing significant rotational excitation. $A_{1}$ resonances thus decay into products with little rotational excitation, in agreement with the result of Fig. 2. On the contrary, for $\bar{A}_{2}$ obtuse isosceles vibrational wave functions, the decay mechanism is an insertion one, where the atom initially close to the principal vertex of the isosceles triangle is pushed toward the two others. This motion provides a bending excitation of the triatom, which turns into rotational energy as the system departs from isosceles configurations, which correspond to an electronic potential crest and a saddle point for collinear geometries. Consequently, fragments arising from $A_{2}$ resonances have significant rotational excitation, as shown in Fig. 2. The strong differences in product-state distributions are therefore the direct consequence of the nodal structure imposed to the vibrational wave function by the geometric phase. A similar nodal structure analysis is used in Ref. [32] to explain the impact of the geometric phase on the cyclic- $\mathrm{N}_{3}$ vibrational spectra.

Similar geometric phase effects are at play in recent coincidence measurements of the energy of the three escaping hydrogens $[11,12]$ after predissociation of various $\mathrm{H}_{3}$ Rydberg states. For the predissociation from the $2 s,{ }^{2} A_{1}^{\prime}$ state in its ground bending mode, most of the available energy is equally shared by two of the three atoms. This corresponds to three lobes with maxima for obtuse isosceles configurations on the Dalitz plots of Refs. [11,12]. Configurations with equal sharing of the energy between the three atoms have a low probability of occurrence which gives a minimum at the center of the Dalitz plot. The situation is opposite for predissociation from the $2 p,{ }^{2} A_{2}$ " state in its ground bending mode: equal sharing of energy between the three atoms now has a high occurrence probability (maximum at the center of the Dalitz plots $[11,12])$. Configurations where one atom carries most of the energy also have a high probability of occurrence and produce secondary lobes with maxima for acute isosceles configurations on the Dalitz plots. These systematic effects do not depend on the choice of the symmetric stretch excitation of the predissociation state and they are equally valid for hydrogen and deuterium.

Our interpretation of these experimental results relies on the identification of the symmetry of the vibrational part of the rovibronic wave function. In the case of Ref. [12], the predissociating $2 s,{ }^{2} A_{1}^{\prime}$ and $2 p,{ }^{2} A_{2}^{\prime \prime}$ states are populated by laser excitation of the $2 p,{ }^{2} A_{2}^{\prime \prime}(l=0, N=0, K=0)$ metastable state followed by a radiative cascade. $N$ is the total angular momentum of the system and $K$ its projection on the $Z$ axis perpendicular to the molecular plane. The initial metastable rovibronic wave function has an $A_{2}$ symmetry (vibrational and rotational contributions are $A_{1}$ and the electronic part is $A_{2}$ ). The rovibronic permutation symmetry $A_{2}$ is conserved in the radiative processes, and Table I gives the symmetries of the resulting $2 s,{ }^{2} A_{1}^{\prime}$ and $2 p,{ }^{2} A_{2}^{\prime \prime}$ states, involving three photons and two-photon radiative cascades, respectively [34].

For both initial states, predissociation occurs mainly on the excited $2^{2} A^{\prime}$ electronic state, which has $\overline{A_{2}}$ symmetry. Indeed, the electronic nonadiabatic couplings of the $2 s,{ }^{2} A_{1}^{\prime}$ and $2 p,{ }^{2} A_{2}^{\prime \prime}$ states to the upper sheet $2{ }^{2} A^{\prime}$ are larger than those to the lower $1^{2} A^{\prime}$ one [35]. The vibrational and rotational symmetries of the final rovibronic wave functions are given in Table I. The $2 s,{ }^{2} A_{1}^{\prime}(l=0 ; N=1,3 ; K=0)$ state decays through vibrational predissociation: its rotational part is unaffected by this decay mechanism; the final vibronic wave function is therefore $A_{1}$ like the initial one, and the final 
electronic and vibrational wave functions are both $\bar{A}_{2}$. The final vibrational wave function therefore has a dominant obtuse isosceles character, giving maxima on the Dalitz plots of Ref. [12] for these configurations, and nodal lines for acute isosceles configurations. These nodal lines intersect at the center of the figure, where the Dalitz plot has a minimum. The $2 p,{ }^{2} A_{2}^{\prime \prime}(l=0 ; N=0,2 ; K=0)$ states decay through rotational predissociation, which corresponds to a Hamiltonian coupling term of the form $N_{X} L_{X}+N_{Y} L_{Y}$ involving total and electronic angular momenta. The symmetry of the vibrational wave function is kept untouched in the process. The geometric phase that appears in the electronic part of the wave function in the transition from the $2 p,{ }^{2} A_{2}^{\prime \prime}$ state to the $2{ }^{2} A^{\prime}$ one is canceled by a similar one in the rotational part of the wave function (Wigner rotation functions with $K= \pm 1$ ). The dissociative wave function therefore has an electronic part with $\bar{A}_{2}$ symmetry, a rotational part with $\bar{A}_{1}$ symmetry, and a vibrational one with $A_{1}$ symmetry. Having no nodal line imposed by symmetry, the vibrational wave function has significant amplitude for equilateral triangular configurations. This corresponds to the maximum for equal sharing of the momentum observed at the center of the $2 p,{ }^{2} A_{2}^{\prime \prime}$ Dalitz plots. The lobes for acute isosceles configurations on the Dalitz plots are also compatible with the $A_{1}$ vibrational symmetry. The strong differences between the $2 p,{ }^{2} A_{2}^{\prime \prime}$ and $2 s,{ }^{2} A_{1}^{\prime}$ Dalitz plots are therefore a direct manifestation of the different symmetries in the two cases, and in particular of the geometric phase which shows up in the final vibrational wave function for the $2 s,{ }^{2} A_{1}^{\prime}$ case.

In this Rapid Communication, we have considered the electronic predissociation of the cone states supported by the $2{ }^{2} A^{\prime}$ electronic state. These states decay to highly excited vibrational and rotational states of $\mathrm{H}_{2}$, and appear as broad resonances in $\mathrm{H}+\mathrm{H}_{2}$ transition probabilities. The geometric phase has a strong effect on predissociation product-state distributions. Similar symmetry effects are at play in the threebody predissociation of the $2 s,{ }^{2} A_{1}^{\prime}$ and $2 p,{ }^{2} A_{2}^{\prime \prime}$ states. The differences between $2 s,{ }^{2} A_{1}^{\prime}$ and $2 p,{ }^{2} A_{2}^{\prime \prime}$ Dalitz plots are primarily due to the presence of the geometric phase in the vibrational function for the $2 s,{ }^{2} A_{1}^{\prime}$ case and thus provide experimental evidence of the effect of the geometric phase in a molecular dynamical process.

This work has been supported in part by CNRS for one of the authors (B.L.) and NSF (Grant No. CHE138091). The authors are grateful to CNRS and NSF for allocations of computing time at IDRIS and SDSC, respectively. They acknowledge useful discussions with Dr. U. Galster.
[1] V. Kokoouline and C. H. Greene, Phys. Rev. A 68, 012703 (2003).

[2] G. Herzberg, J. Chem. Phys. 70, 4806 (1979).

[3] I. Dabrowski and G. Herzberg, Can. J. Phys. 58, 1238 (1980).

[4] G. Herzberg and J. K. G. Watson, Can. J. Phys. 58, 1250 (1980).

[5] G. Herzberg, H. Lew, J. J. Sloan, and J. K. G. Watson, Can. J. Phys. 59, 428 (1981).

[6] G. Herzberg, J. T. Hougen, and J. K. G. Watson, Can. J. Phys. 60, 1261 (1982).

[7] U. Müller and P. C. Cosby, J. Chem. Phys. 105, 3532 (1996).

[8] U. Galster, P. Kaminski, M. Becke, H. Helm, and U. Müller, Eur. Phys. J. D 17, 307 (2001).

[9] U. Galster, U. Müller, and H. Helm, Phys. Rev. Lett. 92, 073002 (2004).

[10] U. Galster, U. Müller, M. Jungen, and H. Helm, Faraday Discuss. 127, 439 (2004).

[11] C. M. Laperle, J. E. Mann, T. G. Clements, and R. E. Continetti, Phys. Rev. Lett. 93, 153202 (2004).

[12] U. Galster, F. Baumgartner, U. Müller, H. Helm, and M. Jungen, Phys. Rev. A 72, 062506 (2005).

[13] R. Porter, R. Stevens, and M. Karplus, J. Chem. Phys. 49, 5163 (1968).

[14] B. Lepetit, Z. Peng, and A. Kuppermann, Chem. Phys. Lett. 166, 572 (1990).

[15] M. V. Berry, Proc. R. Soc. London, Ser. A 392, 45 (1984).

[16] G. Herzberg and H. C. Longuet-Higgins, Discuss. Faraday Soc. 35, 77 (1963).

[17] H. J. Wörner, R. van der Veen, and F. Merkt, Phys. Rev. Lett. 97, 173003 (2006).

[18] J. C. Juanes-Marcos, S. C. Althorpe, and E. Wrede, Science
309, 1227 (2005).

[19] R. Abrol, A. Shaw, A. Kuppermann, and D. Yarkony, J. Chem. Phys. 115, 4640 (2001).

[20] R. Abrol and A. Kuppermann, J. Chem. Phys. 116, 1035 (2001).

[21] A. Kuppermann, J. Phys. Chem. 100, 2621 (1996).

[22] D. Wang and A. Kuppermann, J. Phys. Chem. A 107, 7290 (2003).

[23] A. Kuppermann, Isr. J. Chem. 43, 229 (2003).

[24] F. T. Smith, Phys. Rev. 118, 349 (1960).

[25] A. Kuppermann, J. Phys. Chem. A 110, 809 (2006).

[26] S. Mahapatra and H. Köppel, Phys. Rev. Lett. 81, 3116 (1998).

[27] S. Mahapatra and H. Köppel, J. Chem. Phys. 109, 1721 (1998).

[28] D. Azinovic, R. Bruckmeier, Ch. Wunderlich, H. Figger, G. Theodorakopoulos, and I. D. Petsalakis, Phys. Rev. A 58, 1115 (1998).

[29] S. Mahapatra, H. Köppel, and L. S. Cederbaum, J. Phys. Chem. A 105, 2321 (2001).

[30] B. Jayachander Rao, R. Pabmanaban, and S. Mahapatra, Chem. Phys. 333, 135 (2007).

[31] M. Hamermesh, Group Theory and Its Applications to Physical Problems (Addison-Wesley, Reading, MA, 1962), Chap. 9.

[32] D. Babikov, B. K. Kendrick, P. Zhang, and K. Morokuma, J. Chem. Phys. 122, 044315 (2005).

[33] J. C. Slonczewski, Phys. Rev. 131, 1596 (1963).

[34] U. Galster, Ph.D. thesis, University of Freiburg, 2006, http:// www.freidok.uni-freiburg.de/volltexte/2915/

[35] M. Tashiro and S. Kato, J. Chem. Phys. 117, 2053 (2002). 